\title{
Smart Mirror: A Reflective Interface to Maximize Productivity
}

\author{
Piyush Maheshwari \\ Professor and Head of \\ Engineering \\ Amity University, Dubai
}

\author{
Maninder Jeet Kaur \\ Assistant Professor \\ Department of Engineering \\ Amity University, Dubai
}

\author{
Sarthak Anand \\ Student \\ Department of Computer \\ Science \\ Amity University, Dubai
}

\begin{abstract}
There is no end of objects that could be made "smarter," some being more suited to this than others. Mirrors, for example, provide a large surface ideal for displaying information and interacting with. This paper depicts the design and development of a smart mirror that represents an elegant interface for glancing information for multiple people in a home environment. Face-recognition based authentication is used to detect the user. It provides a webpage based interface to access data feeds and other services. The data feeds use web service based communication to extract data packets available through various APIs offered by websites. All the computing required for this project is done by a Raspberry Pi 3 computer along with a webcam used for face detection and a LCD panel placed behind the mirror to display the interface.
\end{abstract}

\section{Keywords}

Smart Mirror, Raspberry Pi, Facial Recognition, OpenCV.

\section{INTRODUCTION}

This research project has been developed within the context of a time where every day we see more and more connected devices. The Internet transformed our lives by connecting us more easily to information and other people in the virtual world. Mobile phones then became smartphones and since then this concept has erupted and morphed into the Internet of Things, things which connect us to everyday objects. Our research is exploring a new way to use technologies to be more informed. The state of innovation currently is to provide more information, but less interaction to get it. The device that has been researched and designed is called "Smart Mirror". It is a wall mounted mirror which displays relevant items to the user such as weather, scheduling, messages and other fields of interest. The mirror will solve the problems that many people experience every day, getting information without distraction. Before going to bed, the user may want to know whether it will snow the next morning so that they can plan their commute.

The aim of this research project is to explore the impeding shift in how people receive information. We see wall mounted clocks, which provided people with access to the time at a glance. We have displays in airports to show the timetable so that passengers can see the information. We also see massive tickers and advertisements. We have tried to personalize this concept and bring it to the home. The Smart Mirror lets people use their device less, while being presented with more. This is a major step in the evolution of being informed. This is the moment technology is starting to get out of the way. Heads will be up, hands will be set free.

The rest of the paper is organized as below. In Section II, we present some related projects. Section III presents the overview of key hardware and software components of the
Smart Mirror. Section IV discusses the design and architecture of the system. Section V concludes the paper with some discussion on future work.

\section{RELATED WORK}

Our Smart Mirror represents a natural interface that facilitates access to personalized services. This is an attempt to contribute to the design of a smart mirror-like interface as well as the smart environment in which the interface is used for interaction. Below we briefly comment on some related research in this direction. The Smart Mirror contains some devices equipped with a touch screen or TV enhanced externals devices. However, most of them support entertainment and some interactive tasks. The work has been reviewed as follows:

- Mirror 2.0 [1] combines the advantages of a smartphone and a mirror. It contains an LCD display positioned behind the glass. It provides news and weather information and it allows the playback of both videos and music.

- Smart Washbasin [2] displays different information in a washbasin mirror such as mails, weather forecast, the water temperature and pressure, the calendar and the user's weight measured through a built-in-scale in the base portion. The device consists of an Android Tablet that displays the widgets on the basin mirror, made with a semi-reflecting glass put on top of an LCD display. It is possible to control it without touching the screen surface, since it is equipped with proximity sensors able to track the hands position and motion.

- NEOD Framed Mirror TV [3] is a standard LCD screen (up to 50 inches), covered by a mirror, and specifically designed for the screen. The screen provides some TV functionalities, but it does not provide more interactive features.

- Smart Mirror for home environment [4] allows to control all the smart devices at home. It relays on face recognition for authenticating the user and displays personalized information (news, mail, messages etc.). The system exploits a touch screen monitor and two webcams, one for the face recognition and one for the home surveillance.

-Multi Display in Black Mirror [5] by Toshiba is a prototype that combines the functionalities of a tablet together with the reflecting surface of a mirror. It provides two configurations taking into account two different home environments: the bathroom and the kitchen. Considering the bathroom, the prototype provides useful information for the beginning of the day such as the weather forecast and fitness information coming from personal devices. In the kitchen, the setting includes a camera allowing the user to interact through gestures while preparing recipes and controlling the appliances. 
- The Reveal Project [6], created in the New York Times research and development, consists of an LCD Display covered by a mirror glass. The device exploits a Microsoft Kinect for tracking user's movements in real-time. It visualizes different information on its surface (calendar, mail, news, online shopping websites, instant messenger etc.). In addition, it responds to vocal commands. A peculiar feature is the medicine box scanner, which allows the user to buy medicines recognizing their packages.

- Cybertecture Mirror [7], is a complete PC contained into a 37 inches mirror, equipped with a 32 inches LCD screen. Through a smartphone application, the user accesses different information overlaid on the reflected image. The interface allows to visualize instant messages, the calendar, the mailbox, and the weather forecast. In addition, it provides information on the user's physical state. Indeed, the device provides a set of external wireless sensors that allow to measure the user's weight, fat, muscle and bone mass.

- Interactive Mirror [8] by Panasonic seems to be an ordinary mirror: neither camera nor the other sensors suggest the features of a smart object. Once the user sits down in front of it, the mirror displays an enlarged frame for her face, together with menus for accessing different functionalities. The system analyses the face hydration, wrinkles and other details in order to recommend products and treatments to take care of her skin (e.g., to make it softer etc.), to slow ageing and so on. The mirror supports the user in buying such products. In addition, it provides make-up style previews, simulating lighting and ambient conditions (e.g., at home, outdoor, shopping center, etc.).

- Connected Store Demo [9] by eBay and Rebecca Minkoff provides interactive experiences in both the store showcase and in the fitting room. Once finished, the shopper prepares the fitting room with all the items. Inside the fitting room, the user exploits the mirror for looking for other items and/or providing feedback. In addition, she may select some of them for buying.

- Brushing Teeth Mirror [10] displays the information collected by a smart brush about inflammations or infections of the teeth and gums.

- Medical Mirror [11] combines computer vision and signal processing technique for measuring the heart rate from the optical signal reflected of the face. The prototype consists of an LCD display with built-in camera and a two way mirror fitted onto the frame. The smart mirror recognizes the presence of a user when she stands in front of it and, after about 15 seconds, it displays the heart rate below the user's reflected image.

In comparison to works described above, our work is different in that we aimed to develop a working system for providing services in the ambient home environment based on open standards and off-the-shelf technology, where the smart mirror is the interface to access/control various data feeds, information services, and appliances in the environment.

\section{PROPOSED SMART MIRROR}

Please use a 9-point Times Roman font, or other Roman font with serifs, as close as possible in appearance to Times Roman in which these guidelines have been set. The goal is to have a 9-point text, as you see here. Please use sans-serif or non-proportional fonts only for special purposes, such as distinguishing source code text. If Times Roman is not available, try the font named Computer Modern Roman. On a Macintosh, use the font named Times. Right margins should be justified, not ragged. The requirements and specifications of the Smart Mirror took inspiration from people's every day devices that they use including PCs, tablets and smartphones. The mirror integrated similar features from each to give the user what they would expect out of a modern 'smart' device. Figure. 1 is a schematic view of the smart mirror concept. Smart Mirror is a simple mirror that has been enhanced with the help of technology. The aim of the mirror is to provide an easy way for one to access information services such as news feeds, weather, traffic alerts, etc.

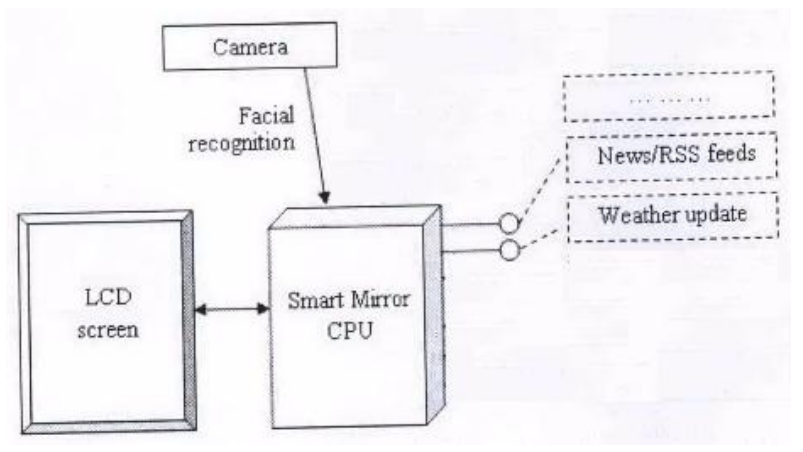

Figure 1. Schematic view of smart mirror

The Smart Mirror CPU is the Raspberry Pi 3 computer. This is where all the software components would lie in. The CPU takes in video information from the camera, runs that information through the facial recognition model and identifies the user. After the CPU knows the person standing in front of it, it would retrieve the information programmed to show for that user. Finally, the CPU projects this information on to the connected LCD Screen.

All these components reside behind a special mirror known as a Two Way Mirror.

\section{A. Hardware components overview}

1) Raspberry Pi

Raspberry Pi is a credit-card sized computer manufactured and designed in the United Kingdom by the Raspberry Pi foundation with the intention of teaching basic computer science to school students and every other person interested in computer hardware, programming and DIY-Do-it Yourself projects.The Raspberry Pi has a Broadcom BCM2837 system on a chip (SoC), which includes 4 ARM Cortex-A53 1.2 GHz cores as the processpr, VideoCore IV GPU and with 1 gigabyte of RAM. It does not include a built-in hard disk or solid-state drive, but it uses a microSD card for booting and persistent storage. It also includes Bluetooh 4.1 Low energy and a 2.4 Ghz 802.11n Wifi [12].The Raspberry Pi is the back bone of this project and is used to fulfill all computational requirements. The Raspberry Pi computer has come out with various versions over the years. Our project employs the use of Raspberry Pi 3 Model B. A microSD card is used to store the operating system and all the software related code for the project.

2) Webcam

A simple USB powered webcam is used to recognize peoples faces.

3) LCD panel

An LCD panel placed behind the mirror is used to present to the user the desired interface. 


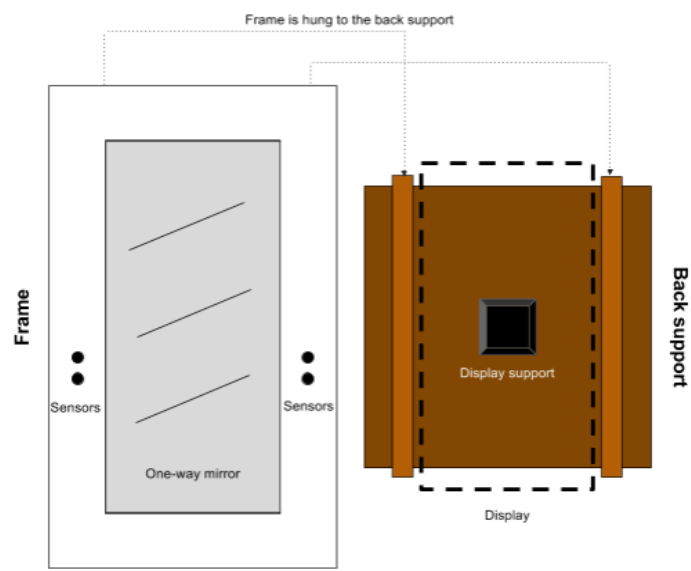

Figure 2: Sketch of the frame hardware design required for Smart Mirror

1) Mirror

A special mirror known as a two way mirror or observation mirror is used in this project. A two mirror is special as compared to an ordinary household mirror. Unlike a household mirror, the two way mirror is not painted with an opaque color on the back, instead its left untouched. This gives the property of the mirror being reflective one side and transparent/translucent from the other. Hence the two way mirror acts as mirror as long as there is no light send from the back of mirror.

\section{2) Frame/Enclosure}

An enclosure is used to cover all the hardware behind the mirror so that the mirror looks as close as possible to a normal mirror as show in the Figure 2.

\section{3) Microphones}

One mode of interaction with the smart mirror is through microphones. Two microphones are used to power the voice recognition capabilities of the device. USB microphones has to be used beacause the Raspberry Pi does not have regular microphone input.

\section{B. Software components overview}

We installed Geany, which is a very lightweight IDE and used it to write all the HTML, Javascript, CSS and Python code.

\section{1) $\mathrm{OpenCV}$}

OpenCV (Open Source Computer Vision Library) is an open source computer vision and machine learning software library. OpenCV was built to provide a common infrastructure for computer vision applications and to accelerate the use of machine perception in the commercial products. Being a BSD-licensed product, OpenCV makes it easy for businesses to utilize and modify the code. The library has more than 2500 optimized algorithms, which includes a comprehensive set of both classic and state-of-the-art computer vision and machine learning algorithms. These algorithms can be used to detect and recognize faces, identify objects, classify human actions in videos, track camera movements, track moving objects etc. OpenCV has more than 47 thousand people of user community and estimated number of downloads exceeding 7 million. The library is used extensively in companies, research groups and by governmental bodies.

It has $\mathrm{C}++, \mathrm{C}$, and Python, Java and MATLAB interfaces and supports Windows, Linux, Android and Mac OS. OpenCV leans mostly towards real-time vision applications and takes advantage of MMX and SSE instructions when available. OpenCV is written natively in $\mathrm{C}++$ and has a templated interface that works seamlessly with STL containers [13]. This project will utilize the facial recognition algorithm from the OpenCV library to recognize users.

2) Raspbian $O S$

Raspbian is a free operating system based on Debian optimized for the Raspberry Pi hardware [14]. Raspbian comes with over 35,000 packages, pre-compiled software bundled in a nice format for easy installation on Raspberry Pi computer.

\section{3) NodeJS}

NodeJS is a javascript engine for server side applications. It comes included with Electron and we used it to launch processes to control things that are not available in web APIs such as the sensors and microphones for voice recognition. We also used it to access the filesystem and read the app files.

\section{4) Python}

Python is a high-level, general purpose, interpreted programming language. Its very popular in the Raspberry Pi community and it has lots of support and libraries. We used it with the microphone to detect sounds and also for control of sensors.

\section{MIRROR DESIGN AND ARCHITECTURE}

Figure 3 shows the overall architecture of the smart mirror's components. The key features of our design are:

Facial Recognition: A webcam placed behind the mirror is used to recognize the user standing in front of the mirror. By recognizing the person, the mirror then knows how to interact or behave next

- Customized user profiles: The output of user recognition then triggers the display of the interface. The interface is designed as per the user. The interface allows a user to view Rich Site Summary (RSS) feeds of social media and email, have access to maps, calendar, weather and time.
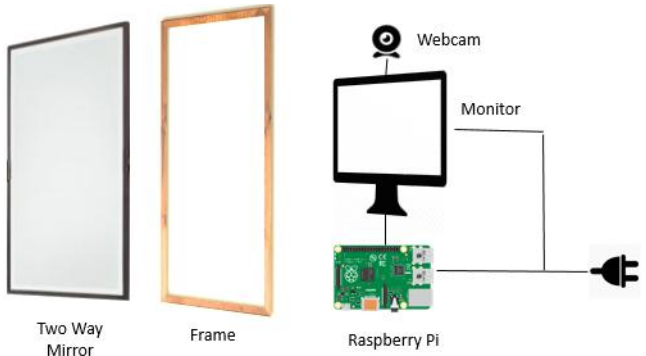

Figure 3. Smart mirror component design and architecture

\section{- Facial Recognition Algorithm}

OpenCV is a popular computer vision library started by Intel in 1999. The cross-platform library sets its focus on real-time image processing and includes patent-free implementations of the latest computer vision algorithms. In 2008 Willow Garage took over support and OpenCV 2.3.1 now comes with a programming interface to $\mathrm{C}, \mathrm{C}++$, Python and Android. OpenCV is released under a BSD license so it is used in academic projects and commercial products alike.

OpenCV 2.4 now comes with the very new FaceRecognizer class for face recognition, so you can start experimenting with face recognition right away. The currently available algorithms are: 


\section{- $\quad$ Eigenfaces (see createEigenFaceRecognizer())}

- Fisherfaces (see createFisherFaceRecognizer())

- Local Binary Patterns Histograms (see createLBPHFaceRecognizer()) [15]

The Smart Mirror uses Local Binary Patterns Histograms algorithm. A more formal description of the LBP operator can be given as:

$$
\operatorname{LBP}\left(x_{c}, y_{c}\right)=\sum_{p=0}^{p-1} 2^{p} s\left(i_{p}-i_{c}\right)
$$

, with $\left(x_{c}, y_{c}\right)$ as central pixel with intensity ; and being the intensity of the neighbour pixel. $\mathbf{S}$ is the sign function defined as:

$$
s(x)= \begin{cases}1 & \text { if } x \geq 0 \\ 0 & \text { else }\end{cases}
$$

This description enables you to capture very fine grained details in images. In fact, the authors were able to compete with state of the art results for texture classification shown in Figure 4. Soon after the operator was published it was noted, that a fixed neighborhood fails to encode details differing in scale. So the operator was extended to use a variable neighborhood. The idea is to align an arbitrary number of neighbors on a circle with a variable radius, which enables to capture the following neighborhoods:

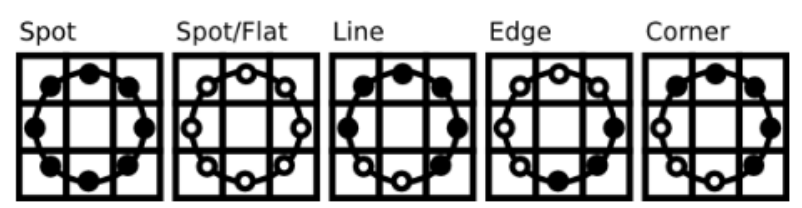

Figure 4: Texture Classification

For a given Point $\left(x_{c}, y_{c}\right)$, the position of the neighbor $\left(x_{p}, y_{p}\right), p \in P$ can be calculated by:

$$
\begin{array}{ll}
x_{p}= & x_{c}+R \cos \left(\frac{2 \pi p}{p}\right) \\
y_{p}= & y_{c}-R \sin \left(\frac{2 \pi p}{p}\right)
\end{array}
$$

Where $\mathrm{R}$ is the radius of the circle and $\mathrm{P}$ is the number of sample points.

The operator is an extension to the original LBP codes, so it's sometimes called Extended LBP (also referred to as Circular LBP). If a point's coordinate on the circle doesn't correspond to image coordinates, the point gets interpolated. Computer science has a bunch of clever interpolation schemes, the OpenCV implementation does a bilinear interpolation:

$$
f(x, y) \approx[1-x \quad x]\left[\begin{array}{ll}
f(0,0) & f(0,1) \\
f(1,0) & f(1,1)
\end{array}\right]\left[\begin{array}{c}
1-y \\
y
\end{array}\right]
$$

By definition the LBP operator is robust against monotonic gray scale transformations. We can easily verify this by looking at the LBP image of an artificially modified image.

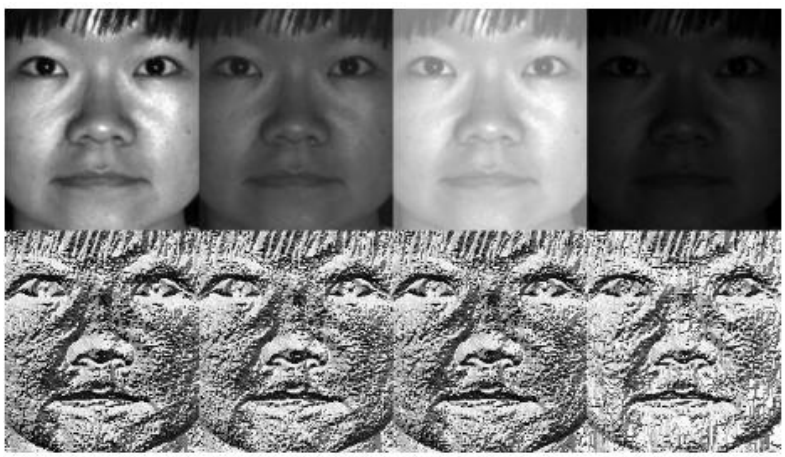

Figure. 5: Test faces

D. Interface Modules

Clock:

Displays clock for set time zone and set hour format

Calendar

Displays the calendar of the current month

Current Weather

Display the current weather of the set location and in the set unit of temperature using OpenWeather API.

Weather Forecast

Displays the weekly weather forecast of the set location and in the set unit of temperature using OpenWeather API.

News Feed

Displays news headlines from the set RSS feed. RSS feeds available are: Gulf News, BBC, CNN and New York Times

Compliments

Displays a compliment appropriate for the time of the day.

Traffic updates

Offers commute time for the set route via Google Maps data.

Facial Recognition

Facial recognition and module swapping based on the current user.

Wunderlist To-Do

Displays Wunderlist todo lists for the set user using Wunderlist API

Quotes

Random daily quotes

Holidays

Displays a list of holidays for the set country using data retrieved from www.calendarlabs.com

\section{PERFORMANCE EVALUATION}

The final results were very satisfying. The device designed can successfully recognize voice input and hand input. We tried to provide a simple API for developers to perform actions in a consistent way. The API works by making use of the inter process communication (IPC) system provided by Electron. This system enables you to communicate through processes using Javascript. 
Developers can call these functions in Javascript to perform different actions consistently.

MOS.showToast(message, duration) - Shows a small message on the bottom part of the screen during the indicated duration.

MOS.showAlert(title, message, alertld) - Shows an alert message with two options that the user can select using gesture input. The alertld parameter is used to obtain the user's input with the onALertPositiveOption and onAlertNegativeOption callback methods.

MOS.setMicrophoneEnabled(enable) -Setter to enable or disable the microphone's clap detection. Useful for apps that play audio so thet don't trigger the microphone.

MOS.setGestureRecognitionEnabled(Enable) - Setter to enable or disable gesture input. Gesture input is disabled by defaut. Any app that wants to use gesture input must call this function.

\section{MOS.setTitle(title) - Sets the status bar message.}

Voice Recognition feature in the designed Smart Mirror uses an online API made by Google. The API has 50 query a day limit but it is the best one available. To use the API you need to make an HTTP POST request with 20000 bit rate. To integrate the service with the OS we created the following bash script.

\section{\#1/bin/bash}

timeout 3.5 arecord $\neg D$ "plughw:0,0" $\neg q \neg f$ cd $\neg t$ wav | avconv $\neg$ analyzeduration $1 \neg y \neg i \neg \neg$ ar $20000 \neg$ acodec flac file.flac

wget $\neg q \neg U$ "Mozilla/5.0" $\neg \neg$ post $\neg$ file file.flac $\neg \neg$ header "Content $\neg$ Type: audio/x $\neg$ flac; $\quad$ rate $=16000 " \neg O \quad \neg$ "http://www.google.com/speech $\neg$ api/v2/recognize?lang=enus\&client $=$ chromium \&key $=A I$

zaSyCXResRGQcGCQhXChLksKdsOOBN7NO_aH8"

>out.json cat out.json

rm file.flac

The above script records a 3.5 second sound file using the microphone, converts it to FLAC, sends it to the API and then prints the result as a JSON string to a file. This was done using a separate script instead of doing it in JavaScript because access to the Microphone using the Web API is not possible in the Raspberry Pi.

The designed Smart Mirror boots on top if Raspbian. To achieve this we modified the Raspbian boot sequence as it immediately starts Smart Mirror after booting. After the initialization, the voice recognition service, socket server are all started. Then the software looks for all the installed softwares or apps any in a folder and it starts the default home screen.

Once the home screen comes, the OS waits for the user input through the voice input service or the socket server. After a user sends a query through one of the possible inputs, the OS processes it and decides which application to open based on the keywords defined by the user.

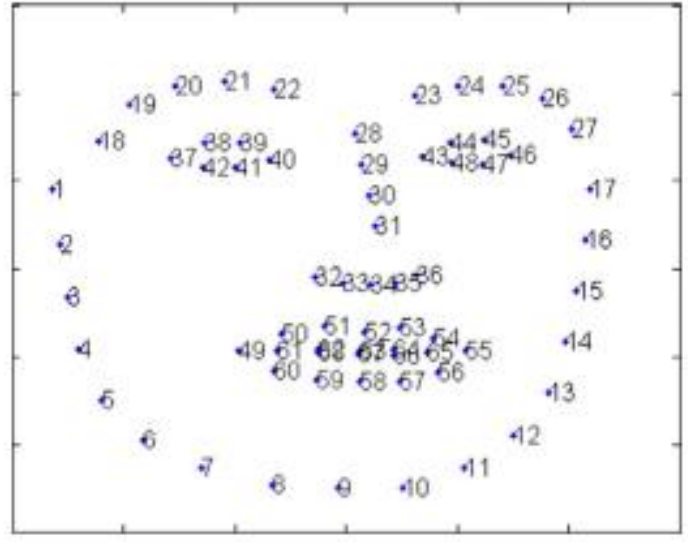

Figure 6: Spacial distribution of landmarks on human face

The algorithm starts with the face detected using special distribution of landmarks on the human face as shown in the Figure 5 using the robust face segmentation method. Normally, a face detection technique is used to localize the face centre and select the region of interest for the reconstruction. Also, a depth segmentation method can be as well an easy and fast way to remove from the image those background and body parts which can produce deformations in the face reconstruction. The typical objects removed in this process include: neck, shoulders or objects in the background. In the proposed algorithm the points are transformed using the estimated rigid transformation model and the ICP algorithm. Afterwards, all the points belonging to the model are projected to a depth image using the camera calibration parameters building a depth sparse segmentation.

\section{CONCLUSION}

The Smart Mirror thus accomplishes this, by still being a mirror without all the technology inside it, making it very approachable to use and integrating seamlessly into our lives. The Smart Mirror has scope in the field of IoT and home automation. The Smart Mirror can be connected to the home appliances, mobile devices, etc. which can expand the functionality of the mirror.

The facial recognition technology used can be future enhanced as a means of security. Adding security means that no one can try to access sensitive data that maybe displayed on your mirror via the use of APIs. We believe that the future of the home will be a brilliantly connected ecosystem of smart technology designed to make your life easier, more enjoyable, and efficient. Obviously there are a ton of opportunities in the home for technology integration but a mirror is one of the best places to start.

\section{REFERENCES}

[1] R. Grynkofi,

"Mirror 2.0, http://bathroominnovation.com.au/finalists\#Year2013 Accessed 2016-01-23

[2] C. Seraku, "Smart Washbasin," http://smartwashbasin.seraku.co.jp/english/ Accessed 2017-01-23

[3] NEOD : NEOD Framed Mirror TV. http://www.neod.org/

[4] M. A. Hossain, P. K. Atrey, A. El. Saddik, "Smart Mirror for ambient home environment," 2007.

[5] Toshiba: Toshiba to Unveil Leading -edge Technologies at CES 2014 . 
http://www.toshiba.co.jp/about/press/2014_01/pr0702.ht

[6] B. House, A. Lloyd, M. Zimbalist, "Reveal Project," http://brianhouse.net/works/reveal/

[7] J. "Cybertecture Mirror," http://www.jameslawcybertecture.com/index.php?section $=$ Company

[8] Panasonic: The Future Mirror. http://youtube/2kc9GQYIE

[9] Ebay: Rebecca Minkoff Connected Store Demo. https://youtube.com/watch?v=6G3JIyG_GeY\#t=10

[10] T. Mullins, "Briushing Teeth Mirror," http://wwwdesignboom.com/contest/view.php?contest_p $\mathrm{k}=36 \&$ item_pk $=44258 \& \mathrm{p}=1$
[11] M. Z. Poh, D. McDuff, R. Picard, "A medical mirror for non-contact health monitring," In ACM SIGGRAPH 2011 Emerging Technologies SIGGRAPH '11, New York, NY, USA, ACM (2011) 2:1-2:1.

[12] "What is a Raspberry Pi?" Raspberry Pi What Is a Raspberry Pi Comments. Accessed May 06, 2016. https://www.raspberrypi.org/help/what-is-a-raspberrypi/.

[13] “ABOUT OpenCV,” Accessed May 04, 2016. http://opencv.org/about.html.

[14] "Home" FrontPage. Accessed May 07, 2016. https://www.raspbian.org/

[15] Bouverot, A. The Impact of the Internet of Things: The Connected Home - GSMA. Retrieved May 5, 2016, from http://www.gsma.com/newsroom/wpcontent/uploads/15625-Connected-Living-Report. 